\title{
Relative Intensity Noise Study in the Injection-locked Integrated Electroabsorption Modulator-Lasers
}

\author{
$\underline{\text { Xiaomin Jin }}^{\mathrm{a}}$, Bennet Yun Tarng ${ }^{\mathrm{b}}$, and Shun Lien Chuang ${ }^{\mathrm{b}}$ \\ ${ }^{a}$ Electrical Engineering Department, California Polytechnic State University, \\ San Luis Obispo, CA 93407, USA, phone: 805-7567046,fax: 805-7561458,E-Mail: xjin@calpoly.edu, \\ ${ }^{\mathrm{b}}$ Department of Electrical and Computer Engineering, University of Illinois at Urbana-Champaign, 1406 \\ W. Green Street, Urbana, IL 61801, USA.
}

As low-cost optical transceivers for access network systems, photonic integrated devices are very important. A photonic integrated circuit (PIC) monolithically integrates into one package optical components such as laser diodes (LDs), photodetectors (PDs), passive waveguides, splitters, external modulators, optical amplifiers, wavelength lockers, variable optical attenuators, and wavelength division multiplexing (WDM) filters. Thus, the number of optical alignment components such as lenses and mirrors is significantly reduced, the assembly costs of the module are decreased, and the size of the transceiver module is reduced. In the field of PICs, much effort has been undertaken to study high-speed low-chirp electroabsorption (EA) modulators that can be implemented in transmitters for high-bit-rate long-haul transmission systems. An electroabsorption modulator has commonly been developed monolithically with an integrated DFB laser to eliminate coupling loss at a joint [1]. Our research in this paper focuses on electroabsorption modulator-lasers (EMLs) or sometimes called integrated laser-modulators (ILMs).

Relative intensity noise (RIN), which represents the laser's intrinsic resonance, is a very important property for semiconductor lasers. For optical communication systems, low RIN floor is needed for the transmitter to achieve a desirable signal-to-noise ratio (SNR). The intensity noise spectrum shows a peak near the relaxation frequency, which is an important parameter for the laser system and directly related to the bandwidth of the laser. However, little experimental work on RIN spectra and the variation of the relaxation oscillation frequency for injection-locked semiconductor lasers is available in the literature [2]. Several theoretical simulations of noise characteristics have been reported and have predicted relaxation frequency enhancement with injection locking [3] [4]. In this paper, we report new experimental results and theoretical calculations of RIN spectra of an injection-locked distributed feedback (DFB) laser using an integrated electroabsorption modulator-laser, which has a high reflection (HR) coating on the modulator side and an anti-reflection (AR) coating on the laser facet. This HR/AR coating scheme is exactly opposite to that of normal integrated EMLs used in optical transmitters. In our experiment, as shown in Fig. 1, we use the reverse biased modulator as a photodetector and investigate the injection-locking phenomena of the DFB laser. With the modulators acting as photodetectors, the number of connectors in our setup is reduced, the electrical signal can be directly measured, and a more accurate measurement is obtained. Also, the results for injectionlocked EMLs give us an opportunity to confirm the theories of increased relaxation frequency in injection-locked DFB lasers. As a matter of fact, external optical injection in integrated EMLs is a relatively uninvestigated field. Our presentation is organized as follows. First, we present an improved theory of relative intensity noise of an injection-locked semiconductor laser. Second, the experimental data and theoretical calculation results of RIN of an injection-locked laser using a backward EML are discussed, which are shown in Fig. 2. With injection locking, the noise peak of the system shifts to higher frequency, from $4 \mathrm{GHz}$ to at lease $6 \mathrm{GHz}$. The noise level at low frequency is also lower from $-127 \mathrm{~dB} / \mathrm{Hz}$ to $-130 \mathrm{~dB} / \mathrm{Hz}$. This shows that injection locking is a good candidate for high-speed low noise system application. Our conclusion is summarized at the end. 


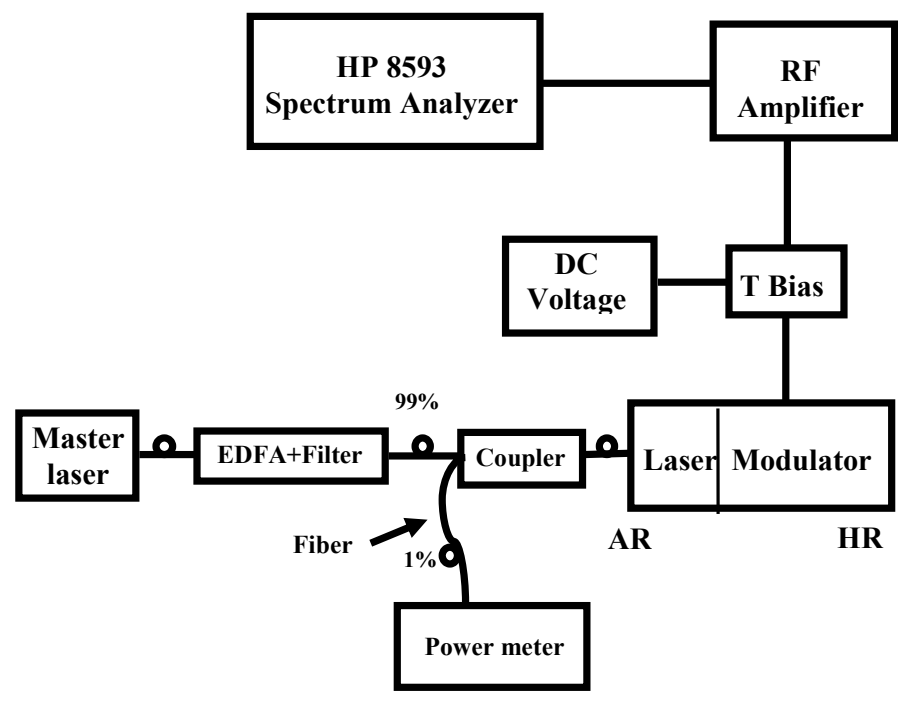

Fig. 1 Experimental setup for RIN measurement of the injection-locked DFB laser using a backward integrated electroabsorption modulator-laser. The pump light from the master laser is injected into the AR facet of the integrated EML, and the modulator is used as a photodetector.
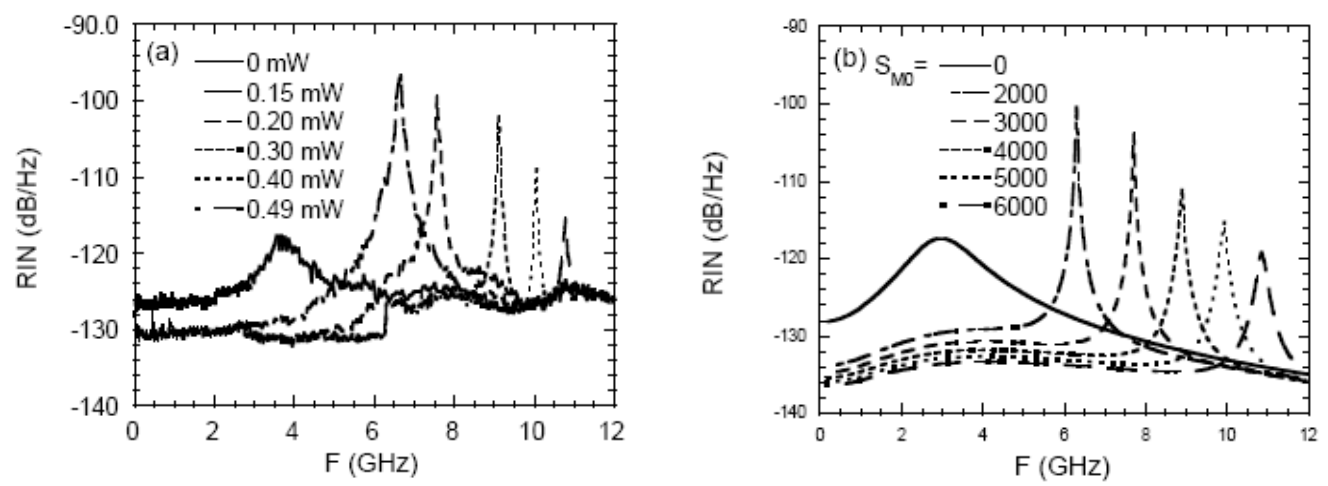

Fig. 2(a) Experimental data and (b) theoretical calculation of RIN spectra of an injection-locked DFB laser system for various pumping powers (or corresponding photon numbers).

\section{References}

[1]T. Tanbun-Ek, L. Adams, G. Nykolak, C. Bethea, R. People, A. Sergent, P. Wisk, P. Sciortino, S. Chu, T. Fullowan, and W. T. Tsang, "Broad-band tunable electroabsorption modulated laser for WDM application," IEEE J. Sel. Top. Quantum Electron., vol. 3, pp. 960-966, 1997.

[2]Y. Hong and K. A. Shore, "Locking characteristics of a side-mode injected semiconductor laser," IEEE J. Quantum Electron., vol. 35, pp. 1713-1717, 1999.

[3]M. C. Espana-Boquera, A. Puerta-Notario, "Noise effects in injection locked laser simulation: Phase jumps and associated spectral components," Electron. Lett., vol. 32, pp. 818-819, 1996.

[4]X. Jin and S. L Chuang, "Bandwidth enhancement of fabry-perot quantum-well lasers by injection-locking," Solid State Electronic, vol. 50, No. 6, pp. 1141-1149, June 2006. 